\title{
Jules-Emile Péan †
}

geb. 29. Nov. 1830 in Chateaudun, gest. 30. Jan. 1898 in Paris.

Erst Tarnier, nun Pean! In ilim verliert die französische Chirurgie den Mann, welcher unbestreitbar den grössten und weitesten Ruf genoss, ohne je einen Lehrstuhl bekleidet zu haben, ja ohne Mitglied der Pariser ,.Société de chirurgie" gewesen zu sein. Die gesamte Chirurgie beherrschend und übend, wie dies seine Ausbildung als Anatoni und Chirurg, als Schüler Nélaton's, als Krankenhaus-Chirurg mit sich brachte, hat Péan von Anbeginn seiner Laufbahn bis zu deren jäheni Abschluss sich mit be-sonderer Vorliebe mit der Chirurgie des Unterleibes und des Sexualorgans beschäftigt. Auf diesem Gebiete hat er seine grössten ïhaten vollbracht, seine unbestrittensten Triumphe errungen, als einer der Schöpfer der modernen Gynäkologie. Péan teilt sich mit Koeberlé in das Verdienst eines Vorkämpfers für die Ovariotomie in Frankreich, indem er, $\Lambda$ venige Jahre nach dem Auftreten von Spencer Wells, durch seine eigenen Erî'olge das besonders durch Nélaton's beharrliches Missgeschick gesunkene Vertrauen in diese grosse Lehrmeisterin der Unterleibs-Chirurgie erweckte, um bald einer ihrer ge suchtesten Meister zu werden. Schon zu jener Zeit gelangen ihm die schwierigsten Bauchoperationen, eine supravaginale Hysterectomie (1866), eine Splenectomie (1867), die erste abdoniinale Totalexstirpation des Uterus und seiner Adnexe (7. Okt. 1869). Mit Koeberlé, der bereits 1863 seine erste supravaginale Hysterectomie mit extraperitonealer Stumpfbehandlung aus-führte, doch diesen hierbald überflügelnd teilt er auch das Verdienst derweiteren Ausbildung dieser Operation mit Hülfe des metallischen Schlingenschnürers. Bekanntlich hat dieselbe auch Porro für die nach ihm benannte Ergäøzung des Kaiserschnittes durch supravapinale Abtragung des puerperalen Uterus als Vorbild gedient. Noch heute gebrauchen einzelne englische Operateure den Serre-noeud. während sonst allerwärts sogar die verbesserte Methode, der extraperitonealen Stumpfbehandlung von He gar-Kalt enbach zu Gunsten der intraperitonealen Verfahren und der abdominalen Totalexstirpation, an deren Ausbildung auch Péan und seine Scliule hervorragenden Anteil nahm, so gut vie verlassen ist.

In die Epochen der technischen Ausbildung der Abtragung des myoma-tosen Uterus fiel die Controverse mit Koeberlé und Verneuil über die

354

Jules-Emile Péan $\dagger$.

Erfindung der hämostatischen Pinzette und der Gefässabklemmung, Forcipressur (1875.) Wenn auch Péan nicht als unbestrittener Sieger aus diesem Streite hervorging, so ist doch die konsequente Vervollkommnung dieses für die ganze Chir $\pi$ rgie äusserst wichtig und fruchtbar gewordenen $\Lambda$ rerfahrens sein Werk *).

Indem es den Operateur von der Aufsuchung der Gefässstämme, der Unter-bindung in der Kontinuität befreite, trug es ungemein viel bei zurVereinfach $\pi n g$. Bequemlichkeit, Raschheit des Operierens und zur Verminderung des Blutver-lustes, aber es drängt doch auch von dem chirurgisch richtigeren Wege ab, die freilich schwierigere, weil exacte Kenntnisse voraussetzende Versorgung der Hauptgefässe vorzunehmen und, indem es auf der einen Seite die Blutstillung er-leichterte, förderte es auf der anderen die Angst vor der Blutung. So ist man denn 
in neuerer Zeit wieder niehr von der präventiven Blutstillung der peripheren Gefässe abgekommen, um sich derjenigen der Gefässstämme, sowie der consecutiven Blutstillung zuzuwenden. Gleichwohl wird sicher die Forcipressur sich für immer daneben behaupten, sowohl als Verfahren der Wahl wie der Nothwendigkeit.

Seine Kühnheit als Operateur bewies Péan aber auch durch Methoden, $\Lambda$ vobei für die Dauer der Operation eine Hämostase nur in beschränktem Masse oder gar nicht stattfinden kann, durch das von ihm ausgebildete Verfahren der Zerstückelung von Geschwülsten, besonders der Uterusmyome, das Morcellement (1887). Anfangs nahm er dasselbe nur vom Abdomen aus vor, wesentlich um die Länge des Bauchschnittes abkürzen zu können, kam aber selbst bald davon zurück. Ein sehr viel weiteres Feld bot ihm bei der Vielgestaltigkeit der Myonie der vaginale $\Lambda$ Veg, auf welchem er Ge-schwülste anzugreifen wagte, deren Sitz und Grösse vordem als unantastbar gait. Mit Recht rühmte er sich, dadurch in sehr vielen Fallen die damals auf ihrer Höhe stehende „Castratio ovarica” umgangen zu haben,

Merkwürdigerweise fand die Hysterectomia vaginalis, welche in Deutschland nach W. A. Freund's kühner That der abdominalen Hyste-rectomie des carcinomatösen Uterus (1878) im Jahre 1881 durch Billroth, Czerny, Schroder ihre Wiedererweckung erfuhr, in dem Vaterlande Récamier's nicht gleich die gebührende Beachtung.

Péan war es, der sich ihr dann zuerst zuwandte und ihr allmählich eine Ausbildung und eine Richtung gab, die dann geradezu ein nationales Gepräge annahm oder wenigstens beanspruchte. Wie natürlich und nahe-liegend bediente sich Péan hierbei seiner Pinces hémostatiques in einer für die Ligamenta lata passenden Form, doch nur, um sie nachher wieder abzunehmen und durch Ligaturen zu ersetzen, ein Verfahren. zu welchem er sich noch im Jahre 1888 für leichtere Fälle von Carcinom und Myom des Uterus bekannte (s. Vorrede zu Secheyron, Traité dHystérotomie et d'Hystérectomie par la voie vaginale). Ein anscheinend kleiner, aberbedeut-samer Schritt war es nun, die Klemmen liegen zu lassen, um sie zur dauernden Blutstillung an Stelle von Ligaturen zu benutzen. Thatsache ist $\Lambda$ vohl, dass Péan mehrere Jahre immer nur das ,,Pincement préventif” übte, und seinem Schûler Richelot erst wird die Idee des ,Pincement definitiv” zugeschrieben. Dem mag so sein. Péan jedenfalls griff diese vordem unge-ahnte Erweiterung des $\Lambda$ Virkungskreises seiner Klemmen mit der ihm eigenen $\Lambda$ Vucht auf und bildete sie, unterstützt durch seine beiden bedeutendsten Schüler Richelot und Segond, zunächst für fíxierte Uteri bei malignen Neubildungen, sowie für grossere myoniatöse Uteri, hier in Verbindung mit 1) In Deutschland werden die hämostatischen Pinzetten allgemein be-zeichnet als Koeb e·rlé'sche Pinzetten. Es ist unbestreitbar, dass Koeberlé ihr Erfinder ist und sich ihrer bereits in den 60 er Jahren in ausgedehntem Massstab bediente. Ja, er wandte sie sogar zuerst als „Pince à demeure" an, indem er sie in einem Fall von Anlegung an die Art. ovarica bis zum Abfallen liegen liess (18G2), in einein anderen Falle bei einem Kaiserschnitt blutende Uteringefässe damit fasste und sie nach 2 Tagen wieder abnahm. (S. Pichevin, Péan et Koeberlé, La Semaine gynéc. 1898, No. 6.) Jules-Emile Péan $\uparrow$.

355

dem Morcellement, weiter aus. Allbekannt sind die bis an die Grenze des Möglichen gehenden, miilisamen Vaginalexstirpationen enormer myomatöser Uteri, an denen Péan und Segond ihre Kraft und ihr Geschick bewiesen. Und jetzt! Wohl in keinem Lande sucht man nun mit solchem Eifer die ab-dominale Totalexstirpation des myomatösen Uterus zu fördern als in Frank-reieh. Péan selbst beteiligte sich noch mit an diesen Bestrebungen. 
Wo ein so weites Gebiet wie das der malignen Neubildungen und der Myome des Uterus fur dessen vagiøale Totalexstirpation gewonnen war, erscheint es uns jetzt naheliegend, dass auch die schweren, besonders eitrigen Adnexerkrankungen, die sogenannten „Beckeneiterungen” dazu herangezogen wurden. Aber gerade um die Frage, wer diesen Schritt zuerst gethan, $\Lambda$ ver diese doch eine besondere Technik erfordernde Operation zuerst vorbedacht durchführte, darüber sind leidenschaftliche Prioritäts-kämpfe geführt worden.

Es muss aber zugestanden werden, dass Péan unabhängig von seinem Nebenbuhler Doyen am 12. Dezember 1887 die erste Operation genannter Art nach vorheriger Diagnose und mit Hülfe von Klemmen ausgeführt habe. Dabei ist mit in Rechnung zu ziehen, dass Péan anfänglich die blosse Wegnahme des Uterus, die „Castration uterine” für genfigend hielt. die vereiterten Adnexe zur Heilung zu bringen.

Aber auch D oy en's Verfahren der „Castration totale” ist ein durchaus selbstständiges und unterschied sich in wesentlichen Punkten von dem-jenigen Péan's. ,Ta Péan hat wenigstens einen Anteil der Priorität der vaginalen Hystero - Salpingo - Oophorectomie bei chronischen und eitrigen Adnexerkrankungen, wenn man von der Nichtverwendung von Klemmen absieht, noch an Leopold abzugeben.

Auf dem Internationalen Medizinischen Kongress in Berlin 1890 war Péan selbst erschienen, um über die ersten (İ0 Fälle von „Castration uterine” und „Castration utéro-ovarienne” ohne Todesfall zu berichten. Auf den internationalen gynäkologischen Kongressen zu Brüssel (1892) und Genf (1894) brachten er und seine Schüler grosse Zahlen dieser Operationen vor. Doch gerade hier halite der Raum wieder von Prioritätsansprüehen und von kritischen Angriffen, denen Péan in unerschütterlicher Ruhe stand hielt.

Der Höhepunkt seines Schaffens war erreicht. In den letzten Jahren widmete er sich wissenschaftlich fast nur noch der Verteidigung seiner Methoden, die er von neuen Verfahren wie der vaginalen Coeliotomie, den conservativen Adnexoperationen, der abdominalen Radicaloperation bekämpft und bedroht sehen musste.

Aus seiner offiziellen Stellung als Krankenhauschirurg, zuletzt 18 Jahre lang am Hospital St Louis, war er, an der gesetzlichen Altersgrenze angelangt, bereits 1892 ausgeschieden. Dafür hatte er aus eigenen Mitteln ein luxuriöses Privatkrankenhaus errichtet , das „Hòpital International”, an welchem er noch in allerletzter Zeit thätig war.

Nach dieser skizzenhaften Schilderung, $\Lambda$ vas Péan in operativer-Be-ziehnng für die grosse Gynäkologie - die kleine existierte kaum für ihn - geleistet, ist der Umfang seiner übrigen chirurgischen Thätigkeit für uns noch aus seinen überaus zahlreichen grösseren und kleineren Scliriftwerken zu bemessen, bei deren Abfassung er sich vielfach jüngerer Hülfskräfte bediente. Von geradezu monumentalem Charakter sind die 9 Bände der „Leçons de clinique chirurgicale”, deren letzter 1895 erschien; dann die 3 mächtigen Bände ..Diagnostic et traitement des tumeurs de l'abdomen et du bassin", 1880-1895. Von historischem lnteresse sind die Monographien über die Péan eigenen Verfahren der Forcipressur, des Morcellement etc. Er wusste treff-liche Mitarbeiter zu finden in Urdy, Secheyron, Baudron u. A, welche mit ihm zusammen oder selbstständig seine Methoden und Resultate in zum Teil umfänglichen Werken verwerteten. Eine Anzahl tüchtiger „Thesen” wurden unter seiner Anleitung geschrieben. Die vielen kleineren Aufsätze von Péan finden sich fast ausschliesslich in der „Gazette des hôpitaux”, die ge-wiss keinen treueren Mitarbeiter besass, wie ihn, s-owie in den „Bulletins de l'Académie de Médecine" nieciérgelegt, deren Mitglied er war.

356 Jules-Emile Péan $†$. 
Einer der jiingsten Schüler von Péan, Bi·ochin, hat in dem ersten lleí’t der „Revue de Gynécvlogie etc." 1898 einen vollständigen Index seiner Arbeiten zusammengestellt, eine Liste von über 90 Nummern.

Das ,le style c'est Thomme” trifft auf Péan besonders gut zu. La-pidar, kantig aber klar und eindringlich ist seine Darstellung. Die vielen Polemiken, die er zu führen hatte, gaben vielen seiner Schriften etwas streitbares. Seine zahlreichen Nebenbuhler, Neider undFeinde Z $\lambda$ vangen ihn vom Anbeginn seiner cliirurgischen Laufbahn zu einem bestiindigen Krieg, den er als Mann von stählernem Charakter, eine wahre Heroënnatur, ohne jemals zu paktieren, aufnalim und durchkämpfte. Auf den ersten Buck musste man in seiner mächtigen, breitschulterigen Gestalt mit dem scharf gezeich-neten energischen Kopf'e eine gebieterische, selbstständige, durehdringende Persönlichkeit erkennen, den kühnen, kaltblütigen, nie verlegenen Beherr-scher des Messers, den in Aktion zu sehen wohl kein nach Paris ge-kommener Chirurg und Gynäkologe versäumte. Nicht alles, $\Lambda$ vas man da erschaute, mochte einem da $\lambda$ voliI gefallen. $\Lambda$ Venn man Péan am Sonnabend im Krankenhaas St. Louis operieren sail, angethan mit Frack, dessen Aermel etwas umgeschlagen wurden, mit $\Lambda$ rorgesteckter weisser Serviette, dicht umdrängt von Assistenten, Wärtern, Zuschauern, über deren Köpfen liinweg die von Herrn Matthieu eben den Etuis entnommenen Instrumente zugereicht warden, da $\Lambda$ vurde dem jungen deutsclien Gynäkologen mit seiner streng antiseptischen Erziehung recht bänglicli zu Mute bei dieser eigenartigen Schaustellung. Und doch führte Péan gern das Wort Antisepsis im Munde und übte sie auch bis zu einem gewissen Grade bei seinen - Privatopera-tioiien. Erst in seinen späteren Lebensjahren schloss er sicli ganz den Lehren der Asepsis an. Mochte dem deutsclien Gynäkologen daneben auch manches Andere auffallen: die Einseitigkeit von Péan's operativer Technik, die übermässige Yerwendung von Klemmen, die Gleichartigkeit der Operation bei Verschieden-heit der Fälle u. a. m., so musste er doch bekennen, dass das seine ihm ganz eigene Art zu operieren sei, und er musste stets die Kunst der Messer-führung als die verständliche Grundlage seines vielgerühmten Glückes rück-haltlos bewun'dern. Yon der grossen Popularität, die Péan genoss, zeugen auch die vielen Anekdvtcn und Legenden aus seinem Leben, wie sie uns die wissenschaftliche und die Tagespresse gebracht haben, von dem Beginn seiner Laufbahn als armer Müllerssohn bis zu dem Glanz seines wahren Weltrufes. Gar manches harte Wort wurde da auch laut über ihn, nicht ohne von solchen, welche die grossen und edlen Eigenschaften seines Charakters besser kannten, entkräftet zu werden. Der Allversöhner Tod, dem er der Schilderung nach in stoischer Ruhe, mit dem Finger am Pulse entgegensah, wird auch an Péan's Leben die Schatten verklären lassen durch das starke und reine Licht seiner grossen und unvergänglichen Thaten fur Wissenschaft und Menschheit. S anger.

Im Anschluss an diese Schilderung Péan's nach seiner wissenschaft-lichen Thätigkeit, sei es mir vergönnt, dem Menschen, dem Kollegen ein Wort der Erione $\pi m g$ zu widmen. Als ich 1874 mich Péan vorstellte, nahm er mich im Gegensatz •zu manchen seiner Kollegen grossherzig auf. Liebenswürdig und mitteilsam lud er mich ein, ihn in seiner Thätigkeit im Hospital und in seiner Privatpraxis zu begleiten. Seitdem haben sich unsere Wege oft gekreuzt, wo es aber auch war, stets mitten in der angestrengtesten Thätigkeit in Paris, auf den Kongressen in Berlin, Brüssel und Rom, auf Reisen und im Bann seiner eigenen schönen Hãuslichkeit, gab er sich dem jüngeren Fach-genossen mit ríckhaltloser Offenheit und einem Wohlwollen, das dem Umgang mit dem unermüdlich arbeitenden Mann einen fesselnden, imponierenden und dabei 
erwärmenden Reiz verlieh. Seine Kenntnis und Wertschätzung der deutschen Litteratur war nicht gering, obwohl ich ihn nie ein deutsches Wort sprechen gehört habe.

Gern liess er sich über den Stand dieser oder jener Frage berichten, nie kam, nach dem erbittertsten Redekampf in der Sitzung, ein persönlich ver-letzendes Wort auch im trauten Gespräch über seine Lippen. Unerschöpflich $\Lambda$ var er, $\Lambda$ venn das Gespräch auf die ihn zur Zeit beschäftigenden Methoden kam, urn seine Zuhörer zu überzeugen und zu seiner Anschauung herüberzuziehen.

Tagesnachrichten und Notizen.

357

Ueberaus sympathisch gab er sich im Kreise der Seinen, die ihn wie einen Patriarclien verehrten und pflegten. Dann überliess er wohl die Leitung der Unterhaltung seinen Damen, aber manches eingestreute Wort, manche treffende Bemerkung liessen erkennen, welches Verständnis auch der kleineren menschliclien Dinge, welches Interesse für Kimste und schöne Wissenschaften und die Tagesereignisse der auf seiner eigenen Donaäne so rastlose Arbeiter $\mathbf{m}$ sich bewahrt hatte. Uns und vielen unseren Bekannten hat sich Péan nur als gross-denkender feinfühlender Mensch gegeben; als soldier wirdder Altmeister in unserer dankbaren Erinnerung fortleben!

A. Martin.

Tagesnachrichten und Notizen.

Weitere Todesfälle:

Am 16. Januar Dr. Mieh $\beta 1 \beta$ Seibelll Chirurg am Krankenhaus für Unheilbare und zugleich Geburtshelfer an der „Maternità del pio luogo” in Neapel (geboren 1829). Er brachte eine grosse Sammlung enger Becken zusammen und verwertete dieselben in einer geschätzten Monographie. In der Geschichte des Kaiserschnittes ist er bekannt durch Erfindung eines der Pariser Académie de Médecine vorgelegten Instrnmentes, welches dazu bestimmt war, zur Schliessung der Uteruswunde durchgelegte Fadenschleifen von der Uterushöhle her festzuhalten, bis die Wundränder sich geschlossen hätten, das „Stasirafistero”.

Am 15. Februar in Groningen Willem Matthys Hendrik Saenger, weiland Prof, der Geburtshülfe und Gynaekologie daselbst bis zu seinem aus Gesundheitsrücksichten erfolgten Rücktritt im vergangenen Jahre. 1833 zu Bergen op Zoom geboren, promovierte er 1857 in Leiden mit einer Dissertation über Rachitis congenita. Nachdem er sich 10 Jahre lang im Haag der Praxis gewidmet und einen Ruf als tüchtiger Geburtshelfer er-worben hatte, bekam er die Professur in Groningen.

Sein rlauptwerk war ein in mehreren Auflagen erschienenes, vortreff-liches Lehrbuch der Geburtshilfe in holländischer Sprache.

Am 16. Februar, 52 Jahre alt, Dr. Wilhelm MoldenhaußP, a. o.

Professor in der medicinischen Fakultät Leipzig. Er begann seine Laufbahn als Assistent von Credé und veröffentlichte damals neben Casuistiken, einige geburtshülfliche Abhandlungen, die, heute noch von frischem Werte, viel citiert $\Lambda$ verden, $\Lambda$ vie ,.Ein Beitrag zur Lehre vom Pemphigus acutus", ..Ueber das Wesen und die Entstehung der sogenannten Bednar'schen Aphthen", den bekannten Fall von Schwangerschaft in einer einhörnigen Gebärm $\pi$ tter u. a. m. Später wandte sich Moldenhauer dem damals noch jungen Fache der Ohren-, Nasen- und Halskrankheiten zu, in welchem er Hervorragendes , geleistet hat, sowohl als Praktiker wie als akademischer Lehrer, Forscher und Verfasser mehrerer geschätzter Lehrbücher.

Der IV. Band der Verhandlungen der Italienischen Gesellsehaft

für Geburtshülfe und Gynaekologie (19.-21. Oktober 1897) ist eben erschienen. Wir bringen eine Uebersicht der gehaltenen Yorträge am Schlusse des Litteraturverzeichnisses. 
Die Zahl der Mitglieder der Gesellsehaft hat sich vermindert, auf 91, u. a. auch durch Austritt von 4 Mitgliedern, worunter Porro. Eine pietät- 